\title{
NEW FREQUENCY STEP-TUNABLE ECRH SYSTEM FOR ASDEX UPGRADE
}

\author{
D. Wagner ${ }^{1}$, F. Leuterer ${ }^{1}$, A. Manini ${ }^{1}$, F. Monaco ${ }^{1}$, \\ M. Münich ${ }^{1}$, F. Ryter ${ }^{1}$, H. Schütz ${ }^{1}$, H. Zohm ${ }^{1}$, T. Franke ${ }^{1}$, \\ R. Heidinger ${ }^{3}$, M. Thumm ${ }^{2}$, W. Kasparek ${ }^{4}$, G. Gantenbein ${ }^{4}$, \\ A.G. Litvak ${ }^{5}$, L.G. Popov ${ }^{6}$, V.O. Nichiporenko ${ }^{6}$, \\ V.E. Myasnikov ${ }^{6}$, G.G. Denisov ${ }^{5}$, E.M. Tai ${ }^{6}$, \\ E.A. Solyanova ${ }^{6}$, S.A. Malygin ${ }^{6}$
${ }^{1}$ Max-Plank-Institut für Plasmaphysik, EURATOM-IPP, Boltzmannstr.2, D-85748 Garching, Germany e-mail:dietmar.wagner@ipp.mpg.de
${ }^{2}$ Forschungszentrum Karlsruhe,Institut für Hochleistungsimpuls- und Mikrowellentechnik, D-76021 Karlsruhe, Germany
Karlsruhe, Germany Germany Russia
${ }^{5}$ GYCOM Ltd, 46 Ulyanov St., Nizhny Novgorod, 603155, Russia \\ ${ }^{3}$ Forschungszentrum Karlsruhe, Institut für Materialforschung, D-76021 \\ ${ }^{4}$ Institut für Plasmaforschung, Universität Stuttgart, D-70569 Stuttgart, \\ ${ }^{5}$ Institute of Applied Physics, 46 Ulyanov St., Nizhny Novgorod, 603950,
}

\begin{abstract}
A new broadband ECRH (Electron Cyclotron Resonance Heating) system is currently under construction at the ASDEX Upgrade tokamak. This system will employ multi-frequency gyrotrons step-tunable in the range 105-140 GHz. In its final stage the system will consist of 4 gyrotrons with a total power of $4 \mathrm{MW}$ and a pulse length of $10 \mathrm{~s}$. It employs a fast steerable launcher for feedback controlled deposition that allows for poloidal steering of $10^{\circ}$ within $100 \mathrm{~ms}$. Transmission line elements, such as corrugated waveguides, polarizer mirrors and vacuum windows, are designed to cope for this frequency band.
\end{abstract}

Key words: Electron cyclotron resonance heating, step-tunable gyrotron, fast steerable launcher. 


\section{Introduction}

The power deposition in the plasma is primarily determined by the magnetic field. For a single frequency ECRH system this has the consequence that for central heating the magnetic field is no longer a free parameter. However, for plasmas with different plasma currents or different equilibria, the magnetic field should be a free parameter in order to operate at a reasonable edge safety factor $\mathrm{q}(\mathrm{a})$. Furthermore, in a plasma with given parameters, some experimental features, like suppression of neoclassical tearing modes (NTM), require to drive current on the high field side without changing the magnetic field. These requests can be satisfied if the gyrotron frequency is variable. In the experiments performed up to now in ASDEX Upgrade the installed power was only $2 \mathrm{MW}$, of which $1.6 \mathrm{MW}$ was coupled to the plasma. This imposes a limit for current drive, NTM stabilization or generation of internal transport barriers [1]. The requirement for the new system is therefore an installed power of $4 \mathrm{MW}$. Since the current diffusion time in hot plasmas, like those with an internal transport barrier and $\mathrm{T}_{\mathrm{e}}>10 \mathrm{keV}$, is several seconds, we need a pulse duration of $10 \mathrm{~s}$ compatible with the limit of flat top discharges. A further requirement is the capability for very localized power deposition such that its center can be feedback controlled, for instance to keep it on a resonant qsurface. For this purpose fast movable mirrors have been installed.

\section{Gyrotrons}

The first two-frequency GYCOM gyrotron (Fig.1) has been installed and is currently put into operation. It can work at $105 \mathrm{GHz}$ and at $140 \mathrm{GHz}$. The corresponding operating modes are $\mathrm{TE}_{17,6}$ and $\mathrm{TE}_{22,8}$. Here we make use of the $3 \lambda / 2$ and $4 \lambda / 2$ resonances ( $\lambda$ is the wavelength) of the single-disk synthetic diamond vacuum window at these frequencies. A second gyrotron will be steptunable, i.e. it can work at several frequencies within the same frequency range. For each frequency the gyrotron oscillates in a different cavity mode. Fig.2 shows the possible modes and frequencies. Short pulse test gyrotrons have demonstrated efficient generation of power in excess of $1 \mathrm{MW}$ at all these frequencies [2,3]. The frequency can be changed between two ASDEX Upgrade pulses and requires an adjustment of the cryomagnetic field, the gun magnetic field and the collector magnetic field. There is one complicating feature with such a gyrotron: the output beam leaves the gyrotron window at slightly different azimuthal angles and positions so that mirror adjustment in the matching optics unit is necessary. In order to limit the number of required phase correcting mirror sets we will choose 4 frequencies as our main operating modes. 


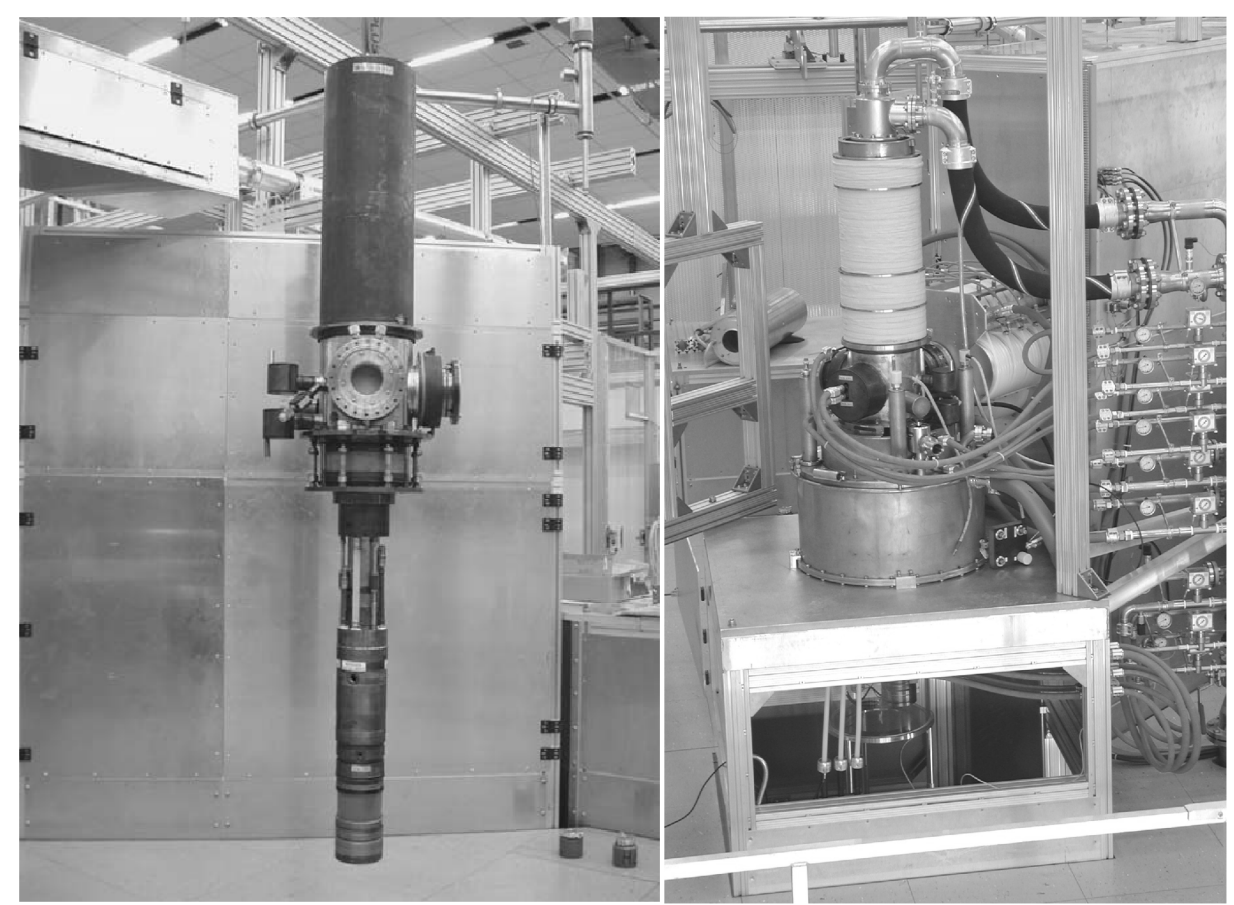

Fig.1 Two-frequency gyrotron Odissey-1 (GYCOM) before (left) and after installation in the magnet (right).

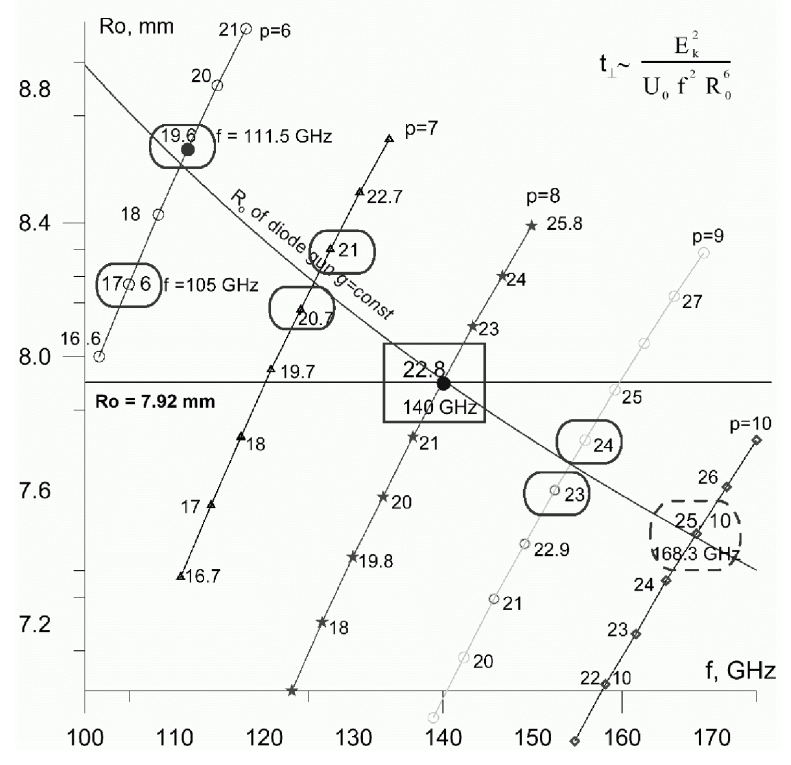

Fig.2 Electron beam radii of diode MIG and caustics for operating modes [3]. 


\section{Power Supplies}

All gyrotrons will have a single-stage depressed collector. Therefore the beam voltage necessary for $1 \mathrm{MW}$ output power can be limited to a maximum value of $60 \mathrm{kV}$. The maximum beam current will be $40 \mathrm{~A}$. Since our power supplies can provide $80 \mathrm{~A}$ at $70 \mathrm{kV}$ we will run 2 gyrotrons from one power supply. There is a separate set of series tetrode and body modulator for each gyrotron (Fig.3), which will allow maximum flexibility for the experimental program.

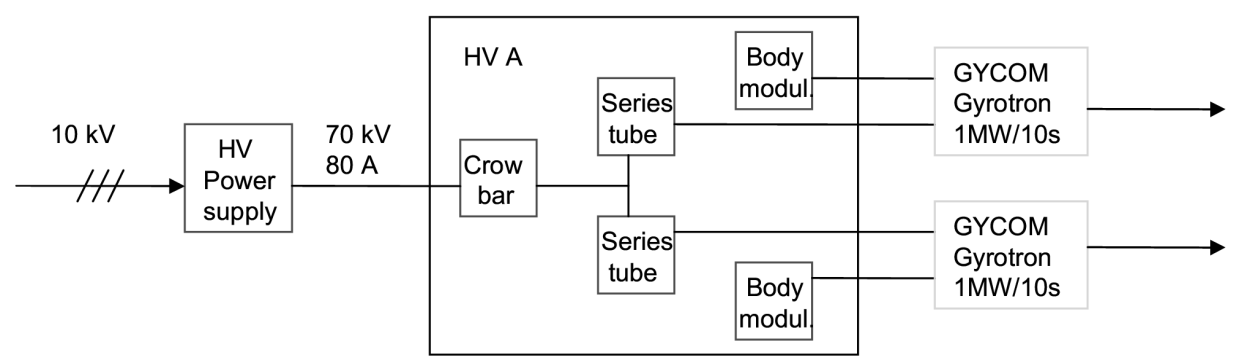

Fig.3 Schematic of power supply and modulator setup.

\section{Matching Optics Unit and Transmission Line}

Fig.4 shows the gyrotron setup with a Matching Optics Unit (MOU) connected to each gyrotron. The MOU (Fig.5) contains different sets of phase correcting mirrors (M1, M2) to match the gyrotron output beam at different frequencies to the transmission line input. These are mounted on rotating discs and can be set according to the operating frequency. The second mirror M2 contains a coupling hole array for pulse monitoring and power measurement. Only one set of polarizers $(\mathrm{P} 1, \mathrm{P} 2)$ with groove depths of $\lambda / 4$ and $\lambda / 8$ scaled to the center frequency of $122.5 \mathrm{GHz}$ proved to be sufficiently broadband to provide the required range of ellipticity and orientation of the polarization ellipse for all necessary injection angles over the whole frequency band of the system [5]. Figure 6 shows the calculated polarisation ellipticity of the output beam of the launcher in dependence of the polarizer angles for three different frequencies. The MOU contains also two switching mirrors that can direct the beam to a short pulse load which is part of each MOU, or to a central long pulse load. The transmission to the torus is in normal air, through corrugated aluminum waveguides with I.D. $=87 \mathrm{~mm}$ over a total length of about $70 \mathrm{~m}$. Since most part of the waveguide path is straight, the number of miter bends could be limited to seven. 


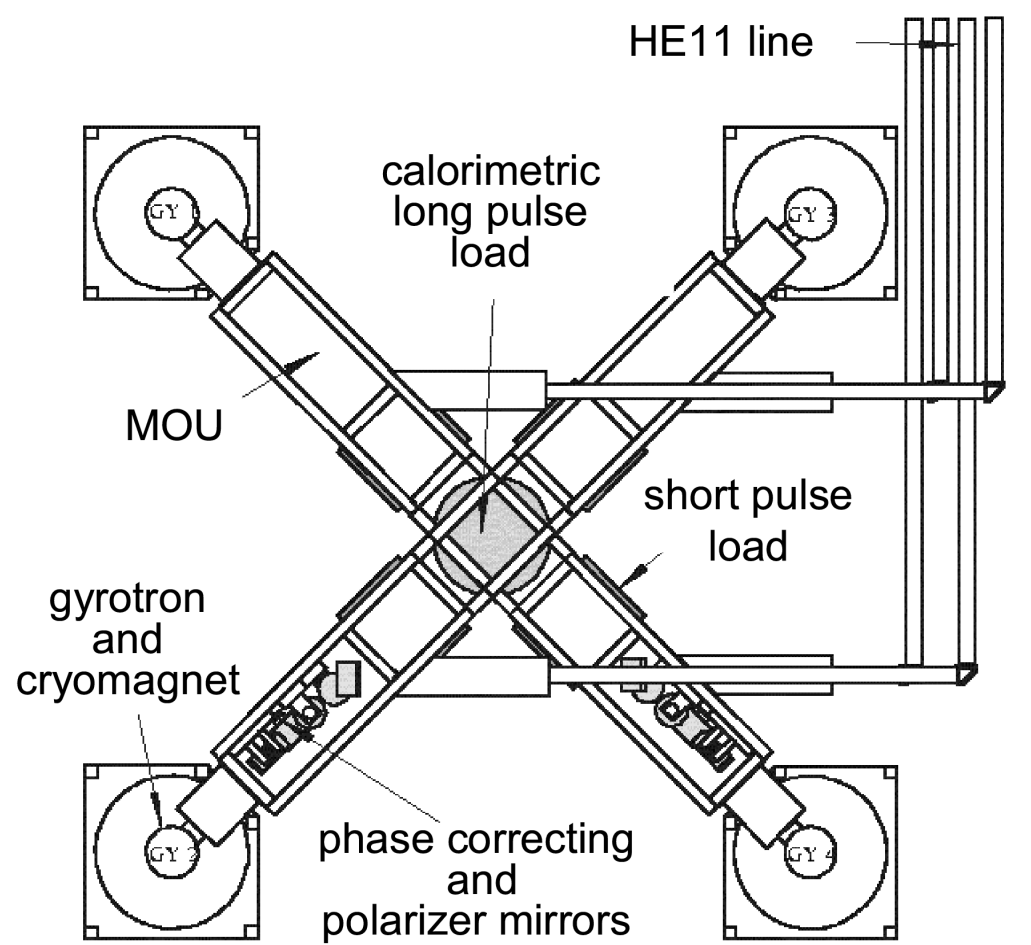

Fig.4 Gyrotron setup with four matching optics units and central long pulse load.

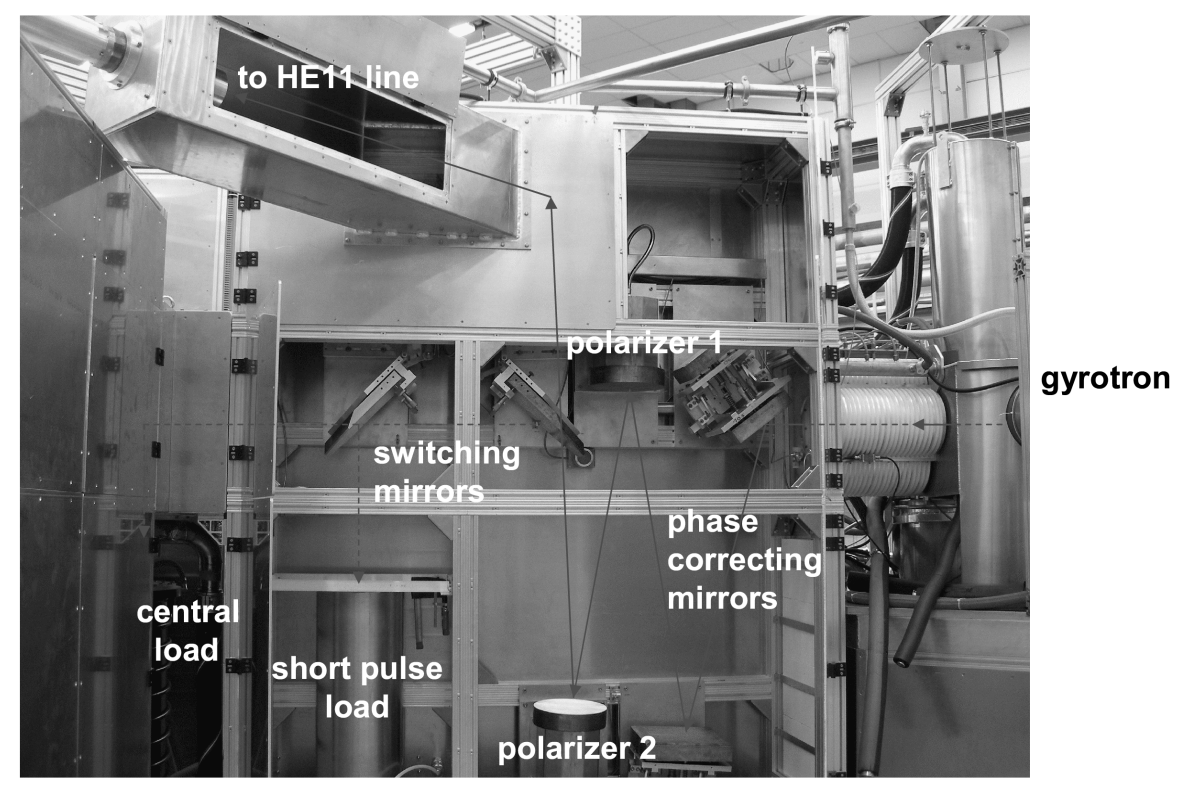

Fig.5 Matching optics unit with phase correcting mirrors and polarizers. 

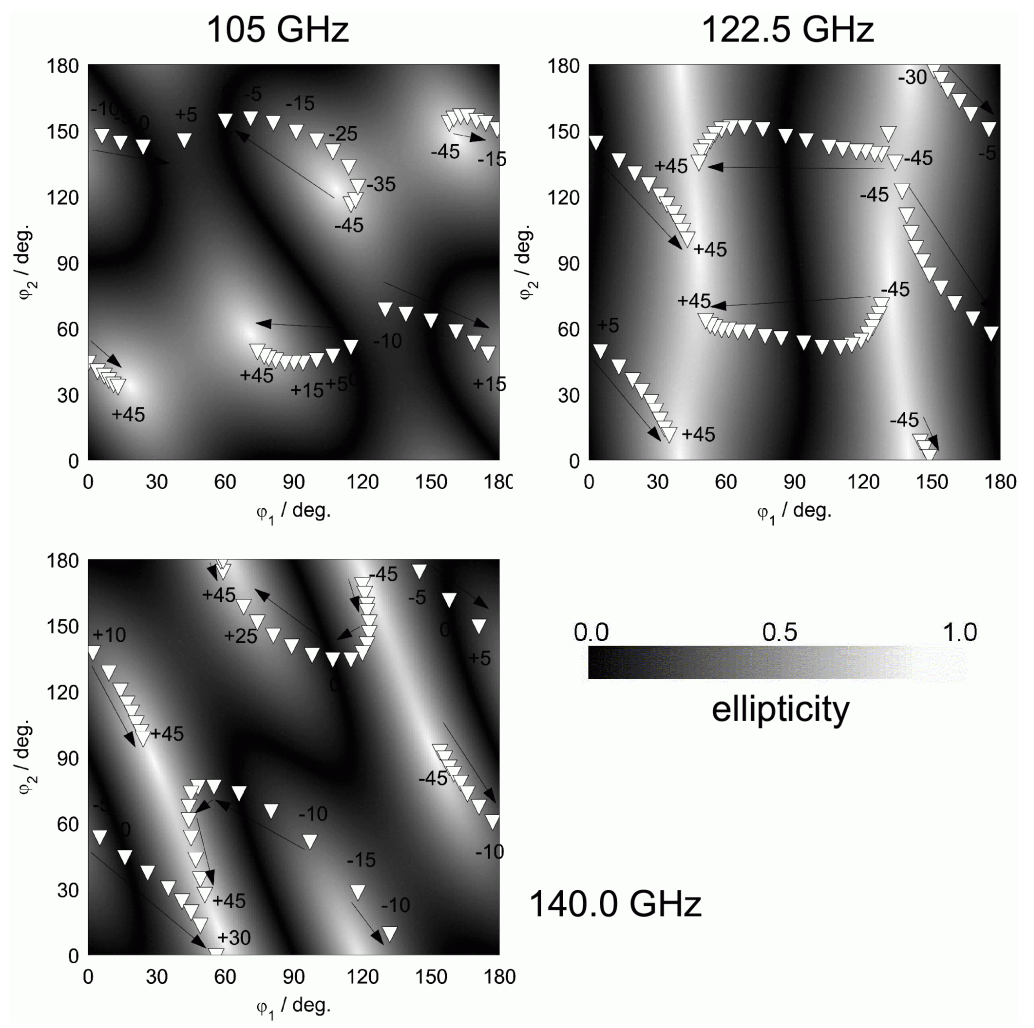

Fig.6 Calculated ellipticity of the broadband two-polarizer set. Indicated by the markers are the required ellipticities for a toroidal angular sweep at a constant poloidal angle of $10^{\circ}\left(\mathrm{B}_{\mathrm{t}}=2.4 \mathrm{~T}, \mathrm{I}_{\mathrm{p}}=800 \mathrm{kA}\right)$.

\section{Broadband Window}

The vacuum windows required for the step-tunable gyrotron and at the torus should be in principle broadband, e.g. a Brewster window. To avoid constraints with respect to polarisation, a tunable double-disk window with a remote controlled adjustment of the distance between the disks will be used at the torus (Fig.7). Two diamond discs with a thickness of $1.8 \mathrm{~mm}$ will be utilized for this window, where the discs are resonant at 105 and $140 \mathrm{GHz}(3 \lambda / 2$ and $4 \lambda / 2$ respectively). For intermediate frequencies the double-disk window can be tuned to a reflection minimum by changing the distance between the two disks. Fig.7 shows the calculated reflection for different distances between the disks. A critical value is the width of the Fabry-Perot resonances at intermediate frequencies between the single-disk resonances. Only a maximum distance of $10 \mathrm{~mm}$ between the disks can be allowed for a possible frequency drift of 100 $\mathrm{MHz}$ during the gyrotron pulse. For the two-frequency gyrotrons a single-disk window is sufficient. 

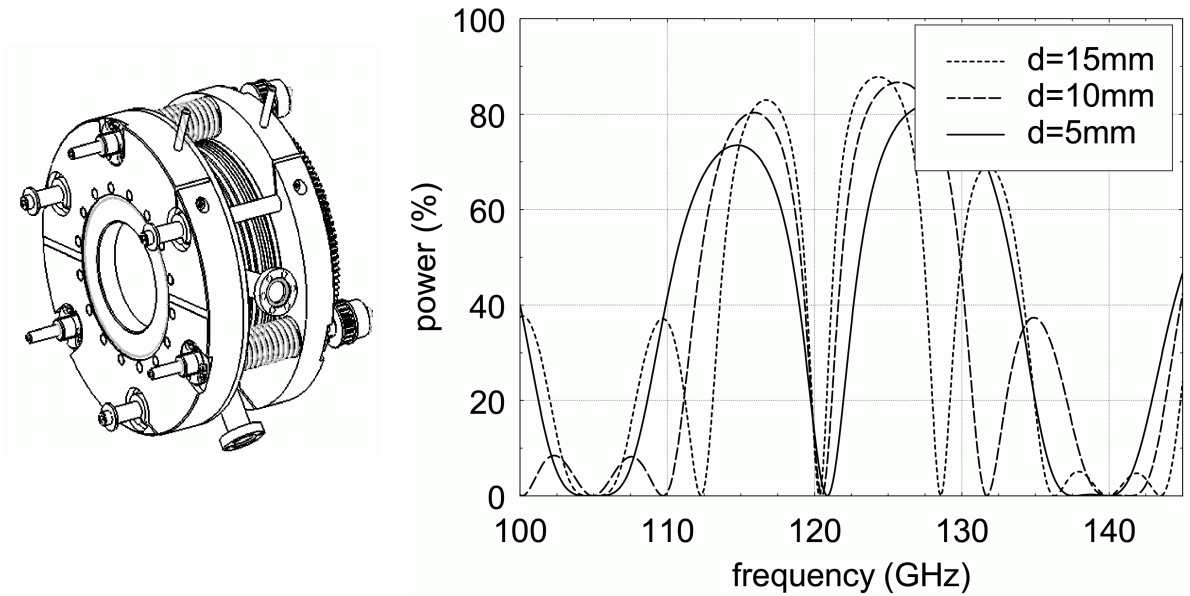

Fig.7 Double-disk window construction (left) and calculated reflection (right) for different distances between the disks.

\section{Fast Steerable Launcher}

A steerable launcher (Fig.8) will enable to steer the beam over the whole plasma cross-section. In order to cope with thermal load, disruption forces and mechanical dynamics of the fast poloidal steering, the mirror is made out of copper plated graphite. Two different types of drives are used for the launcher. A slow drive to rotate the launcher around its axis on a shot to shot basis, mainly to set the toroidal launching angle, and a fast drive to control the poloidal launching angle during a discharge. Fig.9 shows the first two launchers built into ASDEX Upgrade. Fig.10 shows the result of a dynamical test of the launcher during a typical ASDEX Upgrade plasma discharge. The launcher movement (solid line) contains both acceleration and deceleration of the mirror as well as a phase with constant velocity. There is also a delay in the response to the start and stop signal of the remote control (dashed line). The design value of $10^{\circ} / 100 \mathrm{msec}$ for the fast poloidal steering was achieved during the tests. 


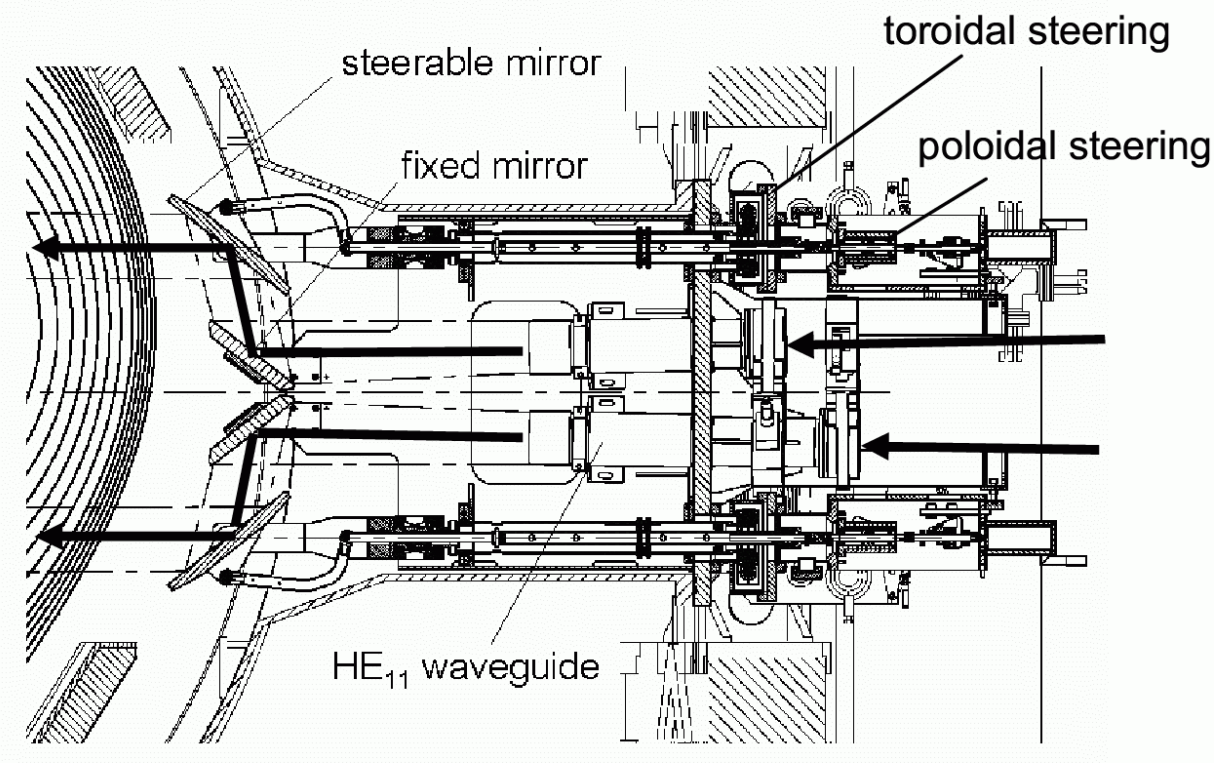

Fig.8 ECRH launcher with a fast spindle drive for fast poloidal steering and a slow worm gear drive to set the toroidal angle.

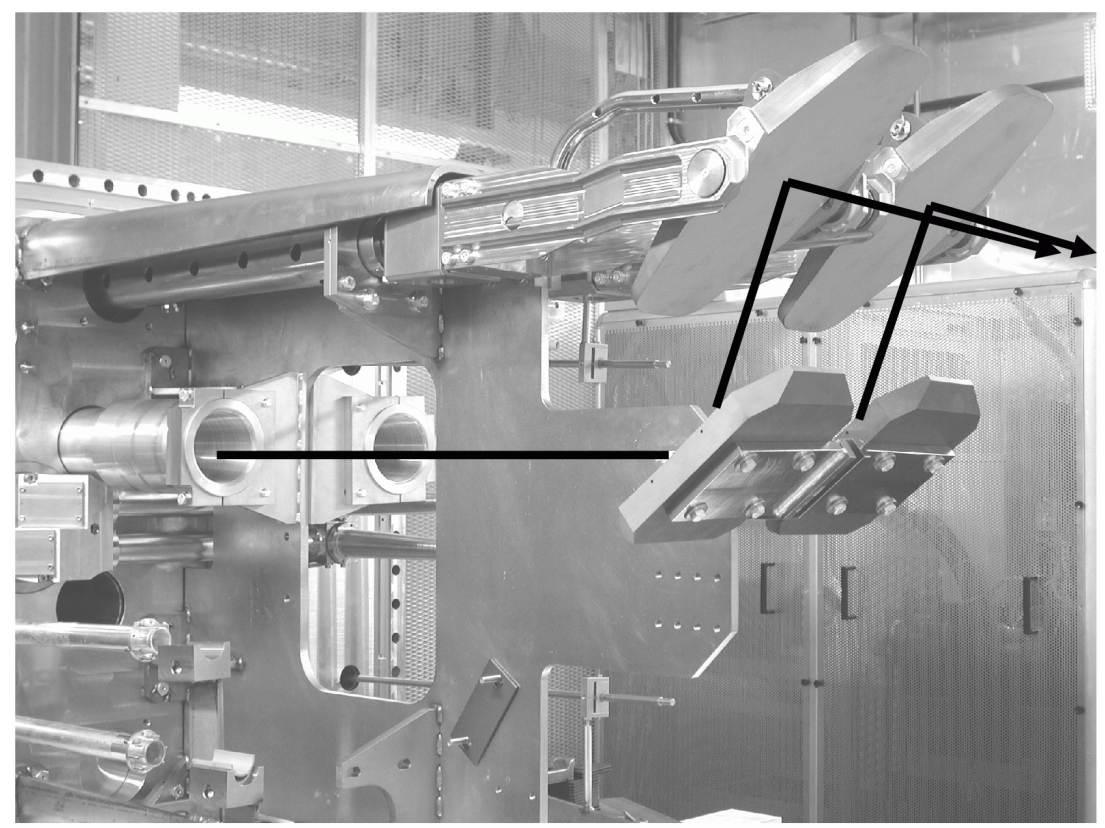

Fig.9 First two launchers built into an ASDEX Upgrade port. 


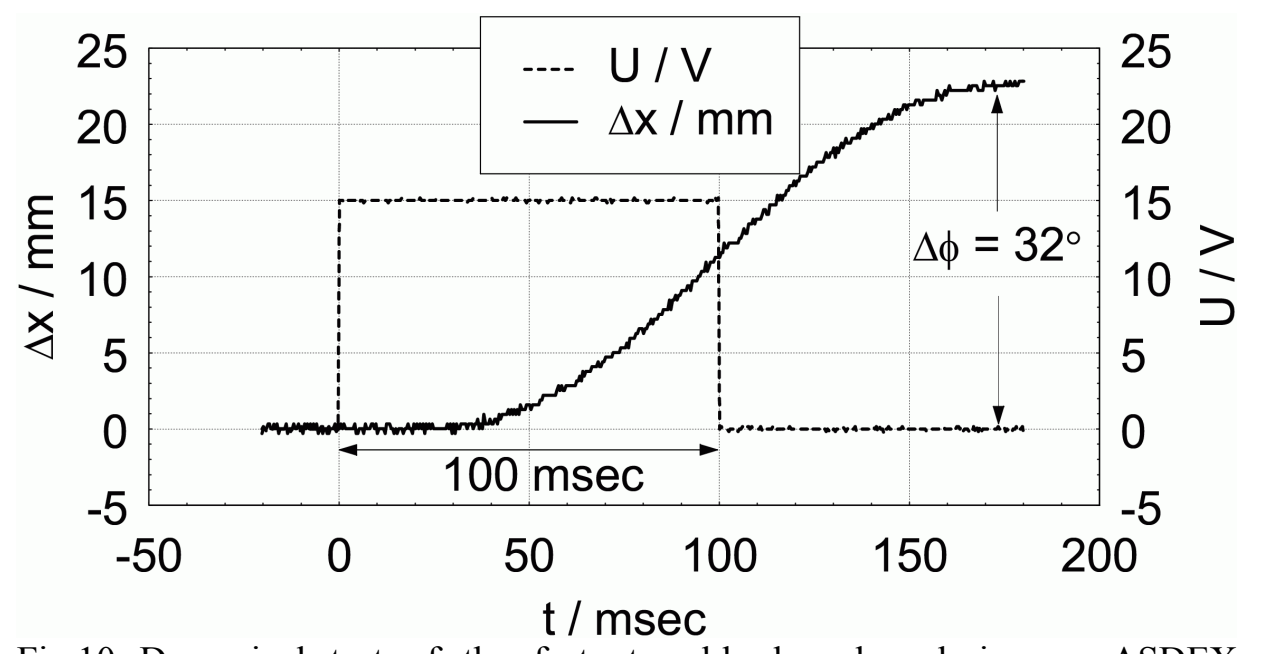

Fig.10 Dynamical test of the fast steerable launcher during an ASDEX Upgrade shot. Dashed line: control voltage, solid line: adjustable stroke of the fast spindle drive.

\section{Summary}

The construction of a new step-tunable ECRH system is in progress at ASDEX Upgrade. The installation of two transmission lines with matching optics units (MOU) for the first two gyrotrons is almost finished. The power supplies for these gyrotrons have been successfully installed and tested. The first twofrequency gyrotron is installed and presently under test.

Factory test results are $900 \mathrm{~kW} / 10 \mathrm{~s} / 140 \mathrm{GHz}$

$700 \mathrm{~kW} / 10 \mathrm{~s} / 105 \mathrm{GHz}$.

\section{Acknowledgement}

This work was carried out within the 'HGF Gemeinsames Forschungszentrum VH-FZ-024: High Power Micro- and Millimeter Waves for Research and Industry'.

\section{References}

[1] Leuterer F., et al., Fus. Eng. Des. 53 (2001) 277

[2] Thumm M., et al., Fus. Eng. Des. 53 (2001) 407

[3] Zapevalov V., et al., Radiopysics and Quantum Electronics 47 (2004) 396

[4] Ellis R., et al., AIP Conf. Proc. 595 (2001) 318

[5] Wagner D. et al., Int. J. on Infrared and Millimeter Waves, 26 (2005) 163

[6] Yang X., et al., Int. J. on Infrared and Millimeter Waves, 24 (2003) 1805 